\title{
DUST DRIVEN WINDS FROM PULSATING STARS
}

\author{
E.A. DORFI, M.U. FEUCHTINGER, S. HÖFNER \\ Institut für Astronomie der Universität Wien, Türkenschanzstr. 17, 1180 \\ Wien, Austria
}

\section{INTRODUCTION}

The cool extended atmospheres of late type giants are sites where dust formation takes place. Radiation pressure on the dust grains is an important force for driving the slow but massive winds observed in such objects. Existing calculations of dust driven stellar winds (e.g. Bowen 1988, Fleischer et al. 1991) suffer from the fact that they include approximations at various levels for different parts of the problem like the hydrodynamics or the dust formation. Furthermore they do not include time-dependent radiative transfer.

In order to overcome these insufficiencies we plan to calculate self-consistent models of dust driven winds with a full description of both the radiation hydrodynamics and the time-dependent dust formation. As a first step, however, we concentrate our investigations on the self-consistent description of the radiation hydrodynamics adopting only a simple description of the dust opacities.

\section{BASIC PHYSICS AND NUMERICAL METHOD}

Intending to calculate the dynamical behaviour of atmospheres of pulsating stars we solve the full system of radiation hydrodynamics including self gravitation and radiative transfer within the Eddington approximation. For the equation of state we assume a perfect gas law $(\gamma=5 / 3, \mu=1.3)$, the Rosseland mean of the gas opacity is set to the constant value of $10^{-4} \mathrm{~g} / \mathrm{cm}^{3}$ and the dust opacity is calculated by the simple formula given in Bowen (1988).

At the inner boundary (situated a few pressure scale heights below the photosphere) the oscillation of the star is simulated by varying the radius sinusoidally. Since we start from an initially hydrostatic atmosphere the radius of the outer boundary at the beginning of the calculations is located close to the photosphere. Therefore we use a Lagrangian boundary in order to follow the expansion of the object to an outer radius of about $5 \cdot 10^{14} \mathrm{~cm}$. At this point we fix the outer radius and employ a simple outflow boundary condition to allow mass loss from the object.

The equations of radiation hydrodynamics are discretized in conservation form on a freely moving coordinate system (adaptive grid). In this context a fixed number of grid points (typically 500) is distributed by simultaneously solving the so called grid equation (Dorfi \& Drury 1987). We employ a monotonic 2nd order advection scheme and the resulting nonlinear algebraic system is solved implicitly. Shock waves are treated by the artificial tensor viscosity (Tscharnuter \& Winkler, 1979) suitable for spherically symmetric problems. 

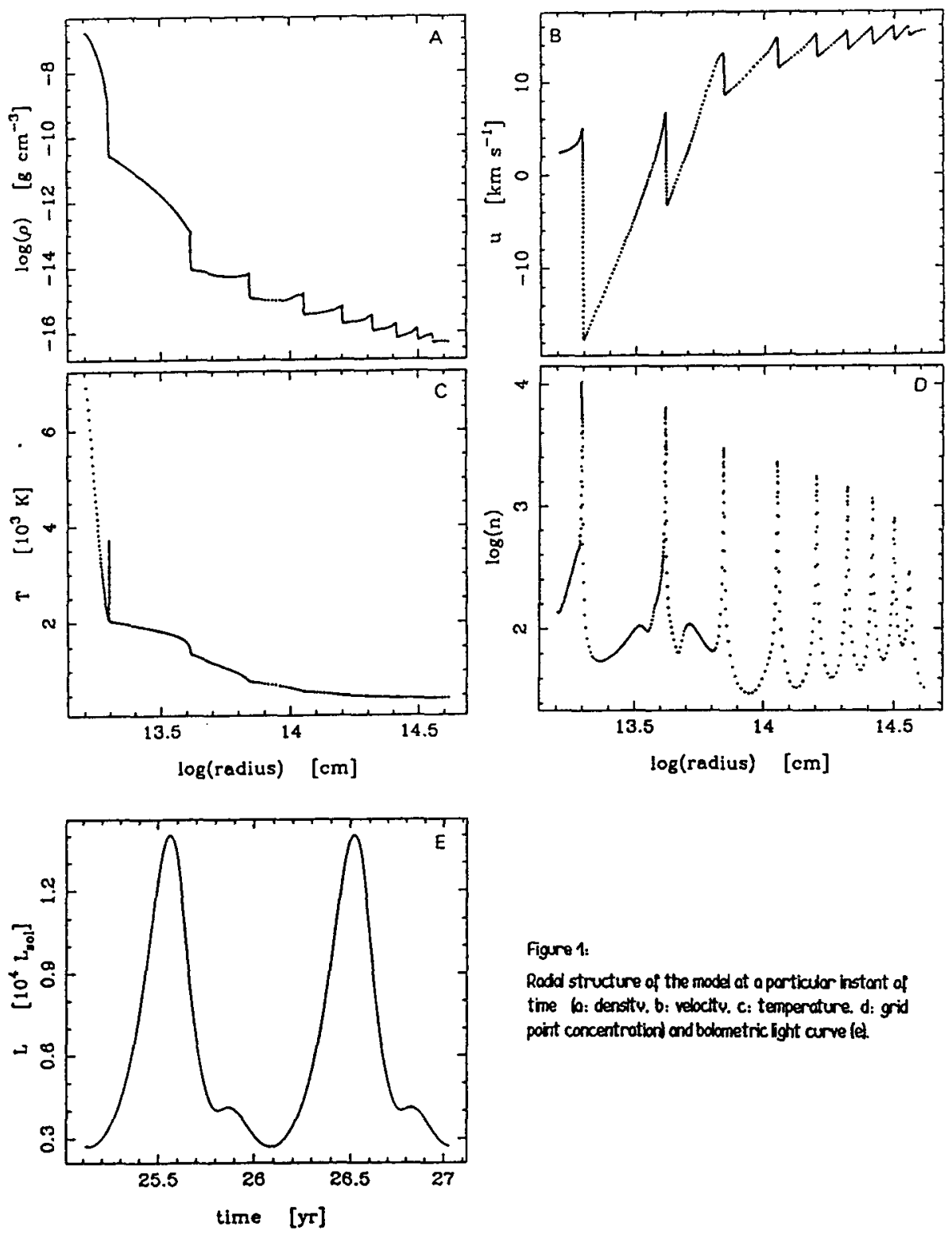

Figure 1:

Rodil structure of the model at a porticulor instont of time b: densitv. b: velocity. c: temperature. d: grid point concentrotioni and bolometric light arve lel. 


\section{RESULTS}

In this section we present a particular model corresponding to a typical Mira variable on the $A G B\left(M=1 M_{\odot}, L=5500 L_{\odot}, T_{\text {eff }}=3000 \mathrm{~K}\right)$. The period of the piston is set to 350 days assuming a velocity amplitude of $3 \mathrm{~km} / \mathrm{s}$.

We follow the evolution until a stationary outflow is reached (after about 30 periods). The radial structure of the physical variables (cf. Fig. 1, a-d) as well as the light curve (cf. Fig. le) are shown in the following plots. The computational improvements can be inferred by comparing these results with the radial structures depicted e.g. in Bowen (1988, Fig. 6-8, calculated for similar initial conditions using a much simpler Lagrangian numerical scheme). In summary we state that the outflow rates $\left(\dot{M}=2.4 \cdot 10^{-6} \mathrm{M}_{\odot} / \mathrm{yr}\right)$ and escape velocities are in the range observed for such pulsating stars.

\section{CONCLUSIONS AND FURTHER INVESTIGATIONS}

A main difference to earlier investigations is the inclusion of time-dependent radiative transfer which e.g. leads to non-sinusoidal light curves (Fig. 1e). In addition it is not necessary to assume adiabatic or isothermal shocks or any kind of cooling functions in order to determine the energy exchange between matter and radiation field. Furthermore Fig. 1 (a-e) clearly demonstrates that a number of unphysical spikes and sawtooth-like features typical for the flow structure of earlier calculations can be avoided by using an accurate numerical method. It is important to note that this method is also suitable for quite different astrophysical situations as e.g. nonlinear stellar pulsations (Dorfi \& Feuchtinger, 1991) or time-dependent dust formation (Dorfi \& Höfner, 1991).

The next step currently under investigation is to couple the radiation hydrodynamics and time-dependent dust formation to construct self-consistent dust driven wind-models of pulsating stars.

\section{ACKNOWLEDGMENTS}

This work is supported by the Österreichischer Fonds zur Förderung der wissenschaftlichen Forschung under project numbers P8411 and P8757.

\section{REFERENCES}

Bowen, G.H.: 1988, ApJ 329, 299

Dorfi, E., Drury, L.O'C.: 1987, Journal of Comp. Phys. 69, 175

Dorf, E.A., Feuchtinger, M.: 1991, A\&A 249, 417

Dorf, E.A., Höfner, S.: 1991, A\&A 248, 105

Fleischer, A.J., Gauger, A., Sedlmayr, E.: 1991, A\&A 242, L1

Tscharnuter, W.M., Winkler, K.-H.A.: 1979, Comp. Phys. Comm. 18, 171 